\title{
Linx
}

Revue des linguistes de l'université Paris X Nanterre

$44 \mid 2001$

Spécificité et histoire des discours sémiotiques

\section{Exploitation de la conception peircienne du signe : un exemple en lexicologie}

Nicole Everaert-Desmedt et Guy Everaert

\section{(2) OpenEdition}

\section{Journals}

Édition électronique

URL : http://journals.openedition.org/linx/1043

DOI : 10.4000/linx.1043

ISSN : 2118-9692

Éditeur

Presses universitaires de Paris Nanterre

\section{Édition imprimée}

Date de publication : 1 juin 2001

Pagination : 95-106

ISSN : 0246-8743

\section{Référence électronique}

Nicole Everaert-Desmedt et Guy Everaert, «Exploitation de la conception peircienne du signe : un exemple en lexicologie », Linx [En ligne], 44 | 2001, mis en ligne le 05 juillet 2012, consulté le 20 avri 2019. URL : http://journals.openedition.org/linx/1043; DOI : 10.4000/linx.1043

Ce document a été généré automatiquement le 20 avril 2019.

Département de Sciences du langage, Université Paris Ouest 


\title{
Exploitation de la conception peircienne du signe: un exemple en lexicologie
}

\author{
Nicole Everaert-Desmedt et Guy Everaert
}

1 Dans cet article, nous partirons de la conception de la théorie sens-texte de Mel'cuk et nous montrerons pourquoi cette conception ne nous satisfait pas entièrement. En effet, la théorie de Mel'cuk traite comme exceptionnelles de nombreuses collocations lexicales. Ce faisant, elle met au rebut des unités qui pourraient pourtant s'avérer révélatrices de la façon dont une langue structure inconsciemment son lexique. Nous proposerons une approche toute différente de la lexicologie et nous essaierons de montrer que cette approche, qui s'inspire de la conception peircienne du signe, pourrait apporter un éclairage original sur le fonctionnement des unités lexicales.

\section{Le traitement des collocations chez Mel'cuk}

2 La lexicologie connait aujourd'hui un renouvellement considérable, dû à l'informatisation des données et à la structure formelle exigée par les applications d'ingénierie linguistique (traduction automatique, interrogation de bases documentaires en langue naturelle, etc.). Parmi les tentatives systématiques de description du lexique, les travaux de Mel'cuk font autorité. Il a en effet proposé une série de préceptes pour décrire le lexique d'une langue ${ }^{1}$. La description essaie de respecter scrupuleusement un certain nombre de principes formels (métalangage systématique et explicite), de cohérence dans le traitement (correspondance entre sémantique et syntaxe), et d'exhaustivité. Elle veut obéir strictement aux règles que se sont données les auteurs pour décrire chacune des unités $\mathrm{du}$ lexique (lexies) et définir l'organisation du dictionnaire (regroupement des lexies apparentées en vocables). On notera particulièrement la richesse des fonctions lexicales dans la description, qui permettent de traiter les synonymes, les antonymes, les emplois métaphoriques, etc. Cette entreprise de description et de systématisation des unités significatives d'une langue est remarquable par sa rigueur et sa précision. 
3 Et pourtant, Mel'cuk et ses collaborateurs reconnaissent eux-mêmes que bien des faits entrent difficilement dans le cadre formel qu'ils se sont imposé, entre autres, ils admettent que les fonctions lexicales n'arrivent pas à traiter correctement certaines données. Citons les auteurs :

Pour être tout à fait honnêtes, nous devons avouer que les fonctions lexicales standard telles que présentées, même en y ajoutant les fonctions lexicales complexes et les configurations de fonctions lexicales, ne couvrent pas totalement l'immense ensemble des cooccurrences lexicales restreintes. Il existe en effet un nombre imprévisible de locutions semi-figées ( = collocations) qui [...] ne peuvent pas être décrites par les fonctions lexicales standard - puisque leur sens est trop spécifique et donc non généralisable.

Un exemple banal permettra de bien saisir le problème. Ainsi, pour dire 'sans ajout de produit laitier' en se référant à un café, un Français dit noir ; cependant, pour le thé, le même sens ne peut pas être exprimé de cette façon: 'thé sans lait' ne s'exprime pas par un thé noir [Mel'cuk et al., 1955 : 150].

Et les auteurs de citer des variantes imprévisibles dans d'autres langues (café solo, caffe macchiatto, caffe latte, etc.). Ils continuent en exprimant leur gêne à propos de ces variantes :

On ne peut les décrire à l'aide d'un formalisme prédéterminé. Le sens de chacune doit être formulé dans le dictionnaire explicatif et combinatoire au coup par coup [ id. : 150].

5 Au coup par coup, disent les auteurs. Cela signifie que lorsqu'on rencontre une collocation, on doit la traiter à part, en la décrivant indépendamment de l'ensemble des structures lexicales. Ainsi, dans l'exemple donné, un étranger ne peut déduire de l'expression « café noir » qu'il s'agit d'un « café sans ajout de produit laitier », surtout s'il n'existe pas dans sa langue maternelle, comme en espagnol ou en italien, une expression du même type. Et, inversement, il est impossible de traduire café noir littéralement par café negro en espagnol. Donc, les auteurs indiqueront, à l'entrée noir ou à l'entrée café, un appendice contenant l'item " café noir ", et sa signification particulière en français : café sans ajout de produit laitier. Et c'est ainsi que l'on peut trouver dans ces travaux des listes du type suivant :

\begin{tabular}{|l|l|}
\hline STEAK, nom masculin & \\
\hline$[\ldots]$ & \\
\hline à peine cuit : & saignant \\
\hline peu cuit : & bleu \\
\hline cuit : & à point (id., p. 1502) \\
\hline
\end{tabular}

Dans cette pratique, toutes les unités du lexique sont donc recensées, mais il faut bien voir - et nous avons vu que les auteurs eux-mêmes en étaient conscients - que le traitement proposé relève d'une procédure d'urgence. Les collocations forment des unités irréductibles au projet lexical formel défini a priori, elles menacent la théorie élaborée, et donc les auteurs les écartent en les stockant dans des listes plus ou moins arbitraires. Cela ressemble à ce que les informaticiens appellent un «patch ». Un patch est quelque chose 
que l'on ajoute après coup, pour corriger des erreurs, des apories ou pour régler un problème pratique. C'est une solution ad hoc, qui n'a aucune portée générale. Littéralement, un patch est un sparadrap, un pansement superficiel, qui tiendra ce que tiennent les sparadraps, l'espace d'un programme... La liste des collocations fonctionne dans la théorie sens-texte comme un patch, à savoir une procédure qui permet de sauver le projet général de description en respectant le critère d'exhaustivité.

7 Peut-on éviter ce genre de patch ? Nous allons voir qu'il est possible de ne pas construire de listes à part, à condition de décider que les unités apparemment irréductibles ne sont pas des scories, mais des éléments à intégrer dans une description d'ensemble. Nous pensons que la théorie générale du signe que l'on trouve chez Peirce pourrait fournir une base pour traiter les collocations.

\section{Traitement des collocations à l'aide des concepts peirciens}

8 La théorie du signe chez Peirce est une théorie générale, impliquant l'ensemble de la culture. Pour un Peircien, les unités du lexique, quelles qu'elles soient, appartiennent à un ensemble cohérent, où les unités s'emboîtent les unes dans les autres à la manière de poupées gigognes. Ainsi, les collocations entrent dans un système lexical, lequel entre à son tour dans un système linguistique, qui fait partie d'un système symbolique plus large, utilisé par des hommes, qui ont des propriétés physiques particulières (ils respirent, dorment, mangent, etc.) et vivent dans un milieu déterminé. Dans cette perspective, les collocations ne sont jamais arbitraires, les emprunts ne sont jamais innocents, ces éléments expriment les tendances profondes et inconscientes qui déterminent une certaine vision du monde. Cette hypothèse lexicale, conforme à la théorie générale du signe chez Peirce, jouera le rôle d'un axiome dans la description des collocations du type « café noir » que nous proposons dans la suite.

9 Si nous passons en revue les emplois les plus courants du mot noir, nous pouvons les classer de la façon suivante :

\begin{tabular}{|l|l|}
\hline$(1 a)$ & chat noir, costume noir. \\
\hline$(1 b)$ & ongles noirs, mur noir de suie. \\
\hline$(2)$ & chambre noire, boîte noire. \\
\hline$(3)$ & race noire, pain noir. \\
\hline$(4)$ & café noir, chocolat noir, savon noir. \\
\hline$(5 a)$ & liste noire, âme noire, jour noir. \\
\hline \hline$(5 b)$ & être noir (ivre), être d'une humeur noire, avoir des idées noires, broyer du noir. \\
\hline$(5 c)$ & marché noir, travailler au noir, magie noire. \\
\hline
\end{tabular}


Ce classement correspond à des emplois différents. En effet :

(1 a et b) Noir décrit l'objet. Il désigne la propriété « surface qui ne reflète pas les rayons lumineux ».

(2) Les expressions désignent des lieux fermés caractérisés par le fait que la lumière n'y pénètre pas. On remarquera que la couleur de l'objet (surface extérieure) n'est guère importante. Par exemple, la boite noire, qui contient l'enregistrement des communications techniques d'un avion, n'est pas noire, mais... orange.

(3) L'utilisation de l'adjectif noir définit des types catégoriels : la race noire s'oppose aux autres « races » et le pain noir s'oppose au pain blanc ou gris.

(4) Dans ces exemples, noir catégorise l'objet et le qualifie positivement : le chocolat noir s'oppose aux autres catégories de chocolat et il est considéré par les amateurs de chocolat comme le plus pur parce qu'il contient le plus haut pourcentage de cacao; le café noir s'oppose au café au lait et il est le plus fort. Un savon noir est un savon puissant qui sert à nettoyer des surfaces. Nous avons isolé les expressions du type (4) parce qu'elles impliquent une valorisation positive.

(5) Par opposition à ce qui précède, ces expressions sont dépréciatives. Dans (5a), noir désigne des entités particulières : la liste noire contient une liste de noms de personnes qui doivent être surveillées de près, une âme noire désigne un vil personnage, un jour noir s'applique à un moment particulièrement néfaste, etc. (5b) contient des états négatifs, propres aux humains (ébriété, humeur, état psychologique) et (5c) caractérise certaines activités humaines illégales par rapport à une norme (sociale ou religieuse). Ainsi faire du marché noir ou travailler au noir sont des activités illicites, et faire de la magie noire est une activité condamnable aux yeux de l'Eglise.

On est frappé de la diversité des emplois : noir permet de décrire, de catégoriser et selon les expressions, il a des effets de sens très différents : tantôt il marque positivement un objet (chocolat noir), tantôt il impose au contraire une vision négative (âme noire, humeur noire, marché noir). Et pourtant, tous les locuteurs ont bien l'impression qu'il s'agit d'une seule et même unité conceptuelle. Comment dès lors rendre compte de cette unité de façon homogène, et expliquer en même temps la diversité ?

Posons le problème en termes peirciens ${ }^{3}$. Un objet dynamique (un segment de réalité) détermine un representamen, par exemple une expression contenant le mot noir (chat noir, boîte noire, ...). Ce representamen renvoie à un objet immédiat (l'un de nos types 1-5), qui constitue un aspect de l'objet dynamique (l'objet auquel l'expression renvoie par l'intermédiaire de l'objet immédiat). Voici deux exemples schématiques :

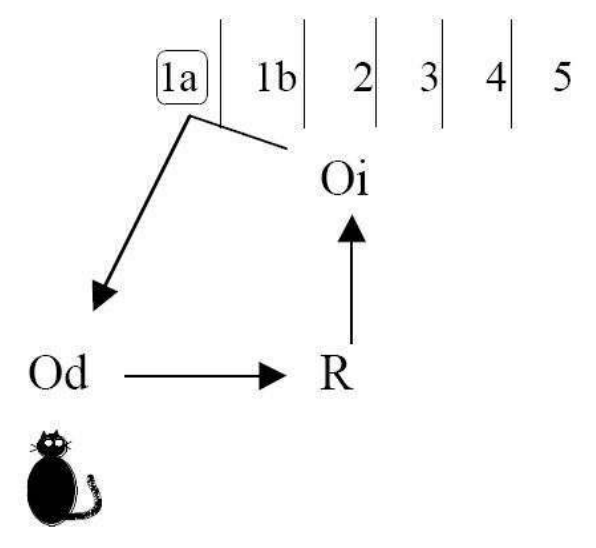




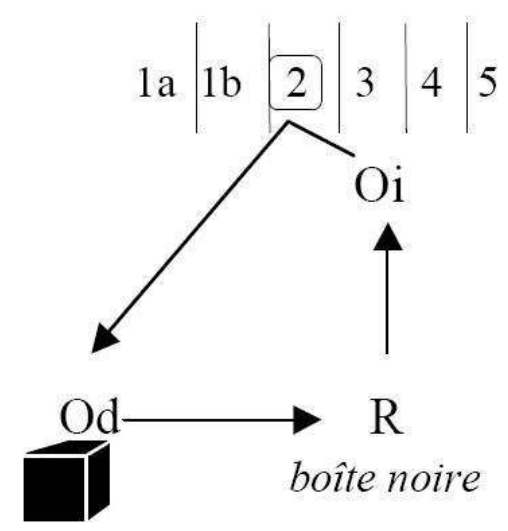

où $\mathrm{R}$ = representamen

Oi = objet immédiat

Od = objet dynamique

12 Ces schémas rendent compte de la diversité des expressions contenant noir, puisque à chaque expression correspond un objet immédiat différent. Cependant les schémas n'expliquent pas l'unité sous-jacente, l'ensemble des utilisations du mot noir. Pour rendre compte de cette unité, nous allons introduire le concept peircien d'interprétant. Ce concept opère la médiation entre le representamen et l'objet immédiat.

13 À ce stade de l'analyse, nous disposons d'un ensemble d'éléments : des representamens (des expressions) et des objets immédiats (les types 1-5). Il nous faut à présent reconstruire l'interprétant, à savoir le principe de constitution de l'ensemble des objets immédiats (à savoir nos 5 types). Nous pouvons donc compléter les schémas précédents comme suit :

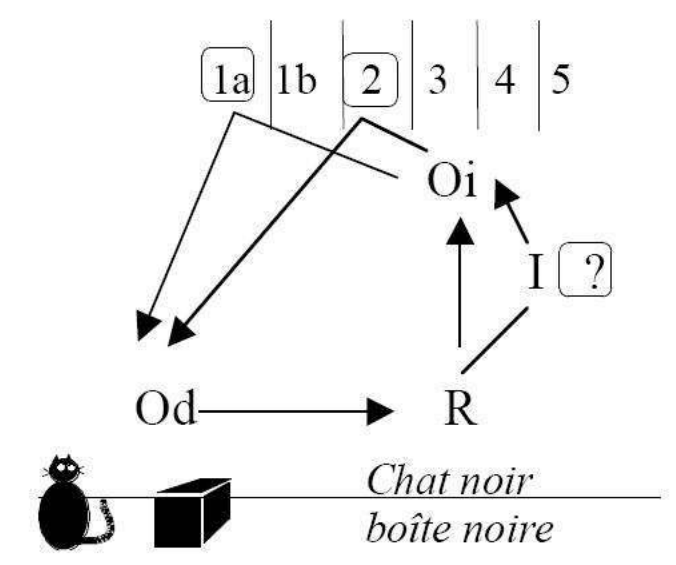

14 Résumons notre démarche : élaboration des objets immédiats (nos types 1-5) par un travail d'observation et de classement des representamens (les expressions) ; à partir des objets immédiats, reconstruction d'un interprétant, qui est une unité abstraite. Les objets immédiats expliquent la diversité des emplois de noir, et l'interprétant expliquera l'unité du signe noir.

Partons d'une première évidence: noir est une couleur, c'est-à-dire une couche qui s'étend sur la surface d'un objet, couche qui a une certaine épaisseur, et qui a comme propriété essentielle de ne pas renvoyer les rayons lumineux. Cela a très bien été exprimé par R. Descartes dans la Dioptrique ${ }^{4}$ : 
[...] il y a des corps qui, étant rencontrés par les rayons de la lumière, les amortissent et leur ôtent toute leur force, à savoir ceux qu'on nomme noirs, lesquels n'ont point d'autre couleur que les ténèbres. pas renvoyer la lumière. Cette description s'applique à des objets concrets qui sont «comptables" ou «massifs". Nous reprenons les critères classiques de divisibilité et d'homogénéité pour opposer les objets comptables et massifs: $\mathrm{x}$ est comptable si, lorsqu'on ajoute un $\mathrm{x}$ à un autre $\mathrm{x}$, on obtient $2 \mathrm{x}$, et si l'on enlève une partie de $\mathrm{x}$, on n'a plus un $\mathrm{x}$, mais autre chose : une boîte plus une boîte font deux boîtes, et si l'on enlève un côté à une boîte, ce n'est plus une boîte. Par contre, si l'on ajoute un peu de sable à du sable, on a toujours du sable (et pas deux sables), et si l'on enlève une partie de sable, on a toujours du sable. Sable est un objet massif par opposition à boite, qui est comptable. Les exemples (4) appartiennent au domaine du massif (café noir, etc), tandis que les autres exemples concrets $(1 \mathrm{a}, 1 \mathrm{~b}, 2)$ appartiennent au domaine du comptable.

Considérons dans un premier temps les exemples $(1 \mathrm{a}, 1 \mathrm{~b}, 2)$ où noir s'applique à des objets comptables. On peut représenter "un objet noir» par le schéma suivant, où la circonférence représente la surface «noire » de l'objet et la flèche représente un rayon lumineux qui n'est pas renvoyé par cette surface :

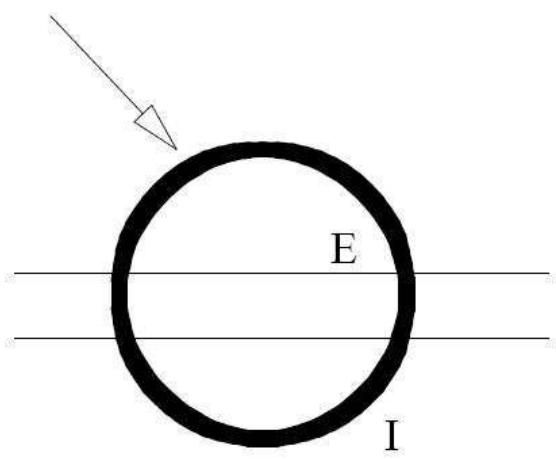

Ce schéma montre que l'objet peut être considéré de deux points de vue : un point de vue extérieur (E), où l'on perçoit une surface noire, et un point de vue intérieur (I), où l'on se trouve dans un espace clos, imperméable à la lumière. Ce double point de vue constitue une opposition fondamentale, à partir de laquelle nous allons poursuivre la construction de l'interprétant.

Considérons tout d'abord le noir extérieur. La couche de couleur noire peut être soit une propriété permanente (exemples 1a, comme chat noir), soit une propriété temporaire (exemples $1 b$, comme ongles noirs).

Lorsque le «noir extérieur » indique une propriété permanente $e^{5}$, il peut servir, si la société en éprouve le besoin, à catégoriser des objets en utilisant l'axe sémantique des couleurs (exemples 3, comme race noire) ${ }^{6}$.

21 Le noir temporaire cache en quelque sorte la propriété naturelle de l'objet - ainsi, des ongles ne sont pas naturellement noirs, mais roses. Le noir temporaire a comme effet de salir, de dénaturer, d'altérer l'essence normale des choses. Ilcontient donc une virtualité négative. Ce trait, issu d'une couche temporaire de noir sur un objet, peut alors être exploité dans un processus de métaphorisation des objets non concrets (exemples (5a) : 
âme noire, jour noir, liste noire), des états psychologiques (exemples (5b) : être noir (ivre), être d'une humeur noire, avoir des idées noires, broyer du noir) ou des activités humaines (exemples (5c) : marché noir, travailler au noir, magie noire), en faisant abstraction du trait «noircitude $»^{7}$.

Examinons à présent le noir intérieur. La clôture délimite un volume interne, caché et protégé, à l'abri de la lumière (exemples 2, comme chambre noire). L'imperméabilité de l'enveloppe assure la qualité du noir : meilleure est l'enveloppe, plus « il fait noir » et plus à l'intérieur le noir est dense ${ }^{8}$. Mais aussi, l'enveloppe protège non seulement de la lumière, mais de toute détérioration. On voit comment on passe à boite noire, qui est un objet destiné à préserver les données qui permettront aux techniciens de comprendre ce qui s'est passé lors d'un crash.

Après les comptables, passons aux objets massifs. Un objet massif se compose de différentes particules. Sa couleur dépend des ingrédients qui le constituent. Le café est naturellement noir, le lait blanc, l'eau transparente ; la farine est blanche, ainsi que le sel, etc. Remarquons que l'on peut ajouter à un objet de ce type d'autres matières, en quantité variable, pour créer un nouveau mélange homogène : on peut ajouter à du café de l'eau, du lait, de l'alcool, de la crème chantilly, du sucre, etc. Notons également que, en cas de mélange, la couleur n'est pas suffisante pour servir de critère de catégorisation : en effet, certaines matières n'affectent pas la couleur du produit final : par exemple, si j'ajoute de l'eau, ou du sucre ou encore un alcool blanc à du café, la couleur noire naturelle ne change guère. Donc, si une société a besoin de nommer un mélange parce qu'il est d'un emploi courant, elle utilisera une expression qui mentionne les ingrédients du mélange ( un café au lait, un café sucré, un crème) ou une métaphore figée (comme cappuccino en italien, café americano en espagnol, ou encore lait russe en français ${ }^{9}$ ).

Considérons ce qui se passe en français. On trouve l'appellation café noir.Elle nous est tellement familière que nous n'en voyons plus l'étrangeté. En effet, il devrait donc suffire pour désigner un café sans ingrédient supplémentaire de dire un café, sans ajouter l'adjectif noir qui est redondant. Si l'on dit un café noir, c'est en fait parce que l'on veut opposer ce type de café aux autres types de café. L'expression est catégorisante. Elle désigne le produit original, et qui s'oppose linguistiquement à toutes les autres catégories de café parce que c'est la seule catégorie mentionnée par la couleur :

\section{café noir $\longleftrightarrow$ café au lait, café sucré, lait russe,}

On a donc, en français, pour catégoriser le café, une opposition primaire qui indique une hiérarchie : le café noir est le produit premier, intégral, qui a une propriété essentielle, sa couleur noire. À partir de ce produit premier, on peut réaliser des mélanges, qui se catégoriseront sur la base des ingrédients ajoutés. Cette opposition primaire n'existe pas dans toutes les langues: ainsi, en italien et en espagnol, on a une seule catégorisation basée uniquement sur les ingrédients, sans hiérarchie :

\section{caffè solo $\Leftrightarrow$ caffè latte $\Leftrightarrow$ caffè machiatto ...}

26 Une fois que l'on a dégagé en français ce principe d'opposition hiérarchique fondamentale ${ }^{10}$, la valorisation peut apparaître : une propriété du produit original - dans notre cas la couleur - est associée à ce qui est intégral et s'oppose ainsi aux mélanges d'ingrédients qui risquent toujours d'altérer l'original. Dans le cas du café, puisque le noir catégorise le café pur, noir contient un trait de valorisation positive, et peut servir pour 
des métaphores positives (association avec la force, la puissance, l'intégralité originale, la virilité, etc.). Et plus c'est noir, densément noir (on parle d'un café serré, bien tassé, etc.), plus la « force » du café sera garantie. Le café noir sera naturellement associé à un champ positif (il réveille, tient éveillé, fait passer la migraine, etc.). Le Robert donne l'exemple suivant, emprunté à R. Queneau :

Un café noir, très noir!

Dans cet énoncé, le premier adjectif catégorise le café (un café sans aucun autre ingrédient), et la reprise de l'adjectif accompagné de l'adverbe très insiste sur l'intensité du noir, c'est-à-dire exprime l'exigence d'un café le plus noir possible, le plus dense possible, donc le plus pur possible, et donc aussi par métaphore, le plus fort. Nous avons ainsi dans le même énoncé à la fois la catégorisation (par opposition aux boissons comportant d'autres ingrédients supplémentaires) et la valorisation. Les marchands de café ont su exploiter cette caractéristique du noir dans leur publicité ou leur marque (cf. les cafés Chat noir). Il en va de même pour le chocolat. Le chocolat noir est le chocolat premier, pur, et d'autant plus pur qu'il est plus noir. Lorsque Côte d'Or présente l'un de ses produits comme Le noir de noir, cette expression catégorise tout d'abord le chocolat comme étant sans mélange, et ensuite elle exploite la valorisation: le noir devient la métaphore de l'excellence.

Résumons notre analyse des expressions contenant le mot noir par le tableau suivant, qui constitue l'interprétant du mot noir :

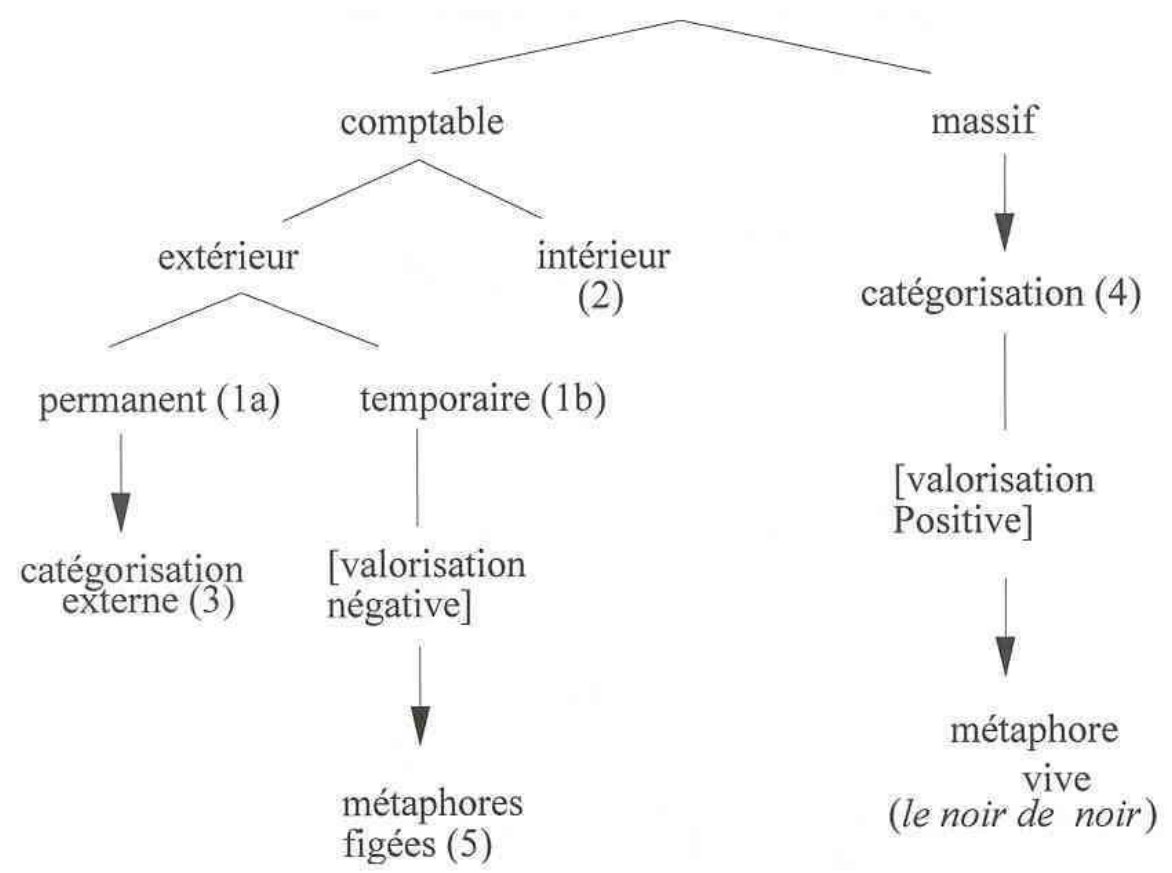

Si nous examinons attentivement le tableau, nous pouvons aisément discerner des éléments de nature différente : la première ligne définit la propriété fondamentale du mot noir. C'est le point de départ de l'organisation de l'interprétant. Cette propriété fondamentale permet de décrire des objets concrets comptables ou massifs, de les caractériser comme ne renvoyant pas la lumière : un chat noir, des ongles noirs ou du café noir ont en commun d'être noirs! La propriété pour un objet d'être noir peut s'appliquer à une propriété ontologique générale pour produire une catégorisation. Les descriptions et les catégorisations peuvent être marquées positivement ou négativement et engendrer 
à leur tour des emplois métaphoriques. Dans le cas de noir, nous constatons des emplois métaphoriques figés (5) sur l'un des parcours de notre tableau (noir $\rightarrow$ comptable $\rightarrow$ extérieur $\rightarrow$ temporaire $\rightarrow$ valorisation négative $\rightarrow$ métaphores figées), et une métaphore vive sur un autre parcours (noir $\rightarrow$ massif $\rightarrow$ catégorisation $\rightarrow$ valorisation positive $\rightarrow$ métaphore vive). Tout est en place, dans ce parcours, pour de nouvelles métaphores figées...

Nous pouvons résumer nos observations comme suit :

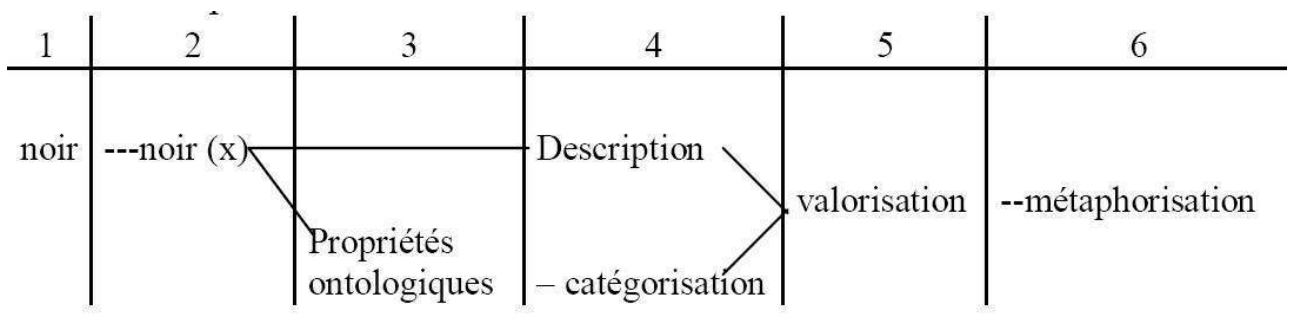

31 La première colonne de ce tableau représente la valeur spécifique de "noir ", qui, appliquée à un objet $x$ (colonne 2), fournit une description (colonne 4). Mais la prédication peut aussi servir d'argument à une propriété ontologique (colonne 3) pour fournir une catégorisation (colonne 4). Description et catégorisation peuvent entraîner une valorisation, positive ou négative (colonne 5), qui sera source de métaphores éventuelles (colonne 6).

L'énonciateur, qui dispose de l'interprétant (savoir sous-jacent du "native speaker »), peut parcourir le tableau à ses différents niveaux d'abstraction pour l'exploiter au mieux de ses intentions communicatives.

Voici un exemple de communication, qui exploite la richesse de l'interprétant de noir. Il s'agit d'un dessin de Faïk intitulé Etat de choc, extrait du magazine La Libre Essentielle ${ }^{11}$. Le dessin est composé de deux vignettes, montrant une femme assise à une table. Dans la première vignette, elle dit, face à un paquet fermé de chocolat Côte d'Or: "Quand je déprime... ». La seconde vignette montre la même femme, face au paquet de chocolat ouvert, croquant à pleines dents une barre de chocolat, et disant : « je broie du noir !». La première image exprime la dépression (état de choc), qui est l'état cognitif négatif porté à son paroxysme dans l'expression broyer du noir - métaphore figée issue de la valorisation négative liée au "noir extérieur temporaire ». L'image de droite montre quelqu'un qui broie littéralement avec les dents du chocolat noir. L'on imagine la suite : après avoir mangé du chocolat noir, expression qui implique une valorisation positive liée à la pureté du produit original, elle aura retrouvé la forme. L'action de broyer littéralement du noir renverse le mouvement négatif de la première image où l'on broyait métaphoriquement du noir, et l'on peut sortir de l'état de choc grâce au choc-olat. L'efficacité de la publicité (et si ce n'est pas une vraie publicité, ce serait une excellente publicité ${ }^{12}$ ) résulte du fait que le produit - le chocolat Côte d'or - est capable de rétablir les déséquilibres psychiques par ses qualités intrinsèques naturelles. Simple, mais terriblement efficace. 


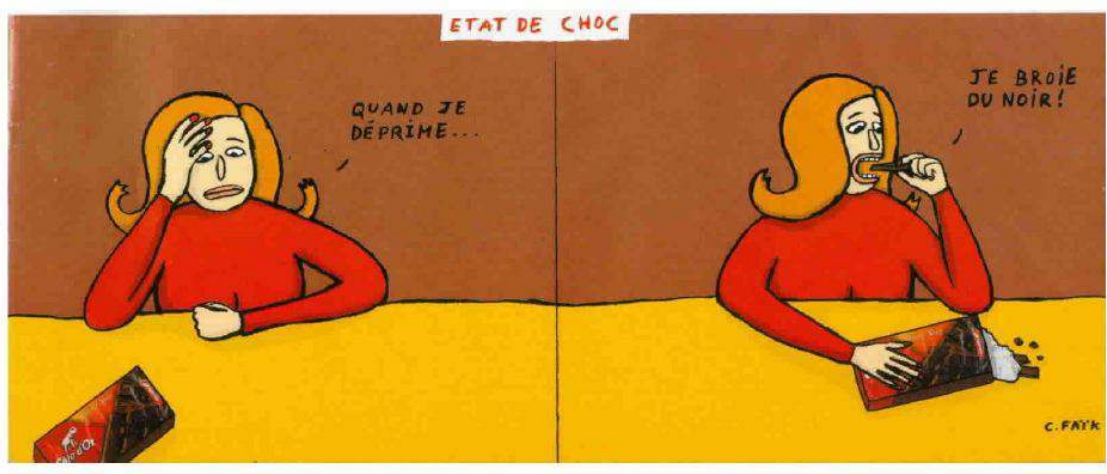

(c) C. Faïk, pour La Libre Essentielle.

\section{Conclusion}

La conception peircienne du signe pourrait se révéler utile pour un linguiste. En effet, elle détermine une approche méthodologique originale : construction d'objets immédiats à partir des representamens et élaboration de l'interprétant. L'examen systématique du lexique selon ces procédures pourrait nous fournir des hypothèses sur la façon dont une langue impose aux locuteurs une certaine vision du monde. Nous pourrions ainsi mieux comprendre les mécanismes à l'œuvre dans la constitution du lexique et son fonctionnement. Les collocations, qui ont servi comme point de départ à notre réflexion, semblent bien être des données fondamentales, qui doivent incontestablement être prises en compte dès le début de la description linguistique.

\section{BIBLIOGRAPHIE}

Everaert-Desmedt N. 1990 : Le processus interprétatif. Introduction à la sémiotique de Ch.S.

Peirce, Liège, Mardaga.

Mel'cuk I. A., Clas A. \& Polguère A. 1995 : Introduction à la lexicologie explicative et combinatoire, Louvain-la-Neuve, Duculot.

Peirce Ch. S. 1931-1935, Collected Papers, vol. 1-6, Cambridge, Massachusetts, Harvard University Press.

- 1958 : Collected Papers, vol. 7-8, Cambridge, Massachusetts, Harvard University Press.

- 1978 : Écrits sur le signe [rassemblés, traduits et commentés par G. Deledalle], Paris, Le Seuil.

- 1982-1995 : Writings of Ch.S. Peirce: A Chronological Edition, Bloomington, Indiana University Press [5 vol. parus].

- 1984 : Textes anti-cartésiens [présentation et traduction de J. Chenu], Paris, Aubier.

- 1992 : The Essential Peirce : Selected Philosophical Writings, Bloomington and Indianapolis, Indiana University Press. 
- 1995 : Le raisonnement et la logique des choses. Les conférences de Cambridge, Paris, Cerf.

- 1998 : The Essential Peirce : Selected Philosophical Writings. Volume 2, Bloomington and Indianapolis, Indiana University Press.

\section{NOTES}

1. Ces préceptes sont présentés dans Mel'cuk et alii [1995].

2. En fait, il faudrait inverser la hiérarchie présentée par Mel'cuk : un steak bleu est moins cuit qu'un steak saignant; il est, selon le Robert Méthodique, «très saignant, presque cru ».

3. Pour une introduction à la sémiotique issue de Peirce, cf. Everaert-Desmedt 1990. Pour une approche plus approfondie, nous renvoyons à la bibliographie où on trouvera les textes essentiels de Peirce.

4. Cité par le Grand Robert à l'article noir.

5. Il n'y a pas de valorisation spécifique au «noir extérieur permanent ». Pour les uns, il est positif (« une limousine noire est plus chic qu'une limousine blanche », « un smoking doit être noir ", « les jeunes aujourd'hui aiment les vêtements noirs », etc. ), pour les autres négatif («une voiture noire est salissante ", "le vrai chic est le smoking ligné », etc.). C'est ce que traduit le dicton : « Des goûts et des couleurs ...»

6. L'expression "pied-noir» s'explique par le mépris à l'égard des anciens colons vivant en Afrique du Nord: une propriété temporaire (avoir les pieds noirs dans le sens de "sales ») a été considérée comme permanente, et est devenue ainsi un critère de catégorisation.

7. Les exemples sont nombreux : un regard noir, un oeil noir, un noir corbeau (où noir n'est pas descriptif, puisqu'un corbeau est toujours noir), etc. Noir est associé à la tristesse, et peut donc symboliser le deuil.

8. Cf. l'expression noir comme dans un four : la qualité première d'un four est d'être fermé pour garder la chaleur. Le four est ainsi un prototype d'espace fermé. On remarquera que l'on emploie l'expression lorsque le locuteur est au centre d'un espace qualifié de noir, et non pas pour qualifier une surface extérieure noire.

9. Certains établissements présentent aujourd'hui à leur carte un Mont-Blanc: il s'agit d'un café surmonté de crème Chantilly, qui se présente comme une petite montagne blanche.

10. Ce principe d'un produit pur, qui s'oppose aux autres, et marqué par une couleur, n'est pas isolé en français. Ainsi, pour reprendre un autre exemple déjà cité de Melc'uk concernant la cuisson du steack, on pourrait faire l'hypothèse d'un découpage basé sur le même principe : le steack bleu s'oppose aux autres présentations : saignant, à point. Le produit premier est marqué par une couleur, ici le bleu, qui marque la pureté originale, comme le montre l'expression sang bleu pour désigner les aristocrates, et les autres présentations culinaires sont désignées par d'autres propriétés. On pourrait ajouter le label blanc-bleu-belge qui désigne la viande produite en Belgique et de qualité garantie. Bleu désigne le prototype de la viande rouge, blanc celui de la viande blanche et belge le lieu d'élevage de la bête. Le fait d'utiliser bleu pour caractériser l'excellence de la «chair » est lié aux veines bleues, plus visibles chez ceux qui ont la peau claire, cette peau claire étant l'indice d'une race pure (cf. le commentaire du Robert Historique à propos de sang bleu).

11. Cet extrait est paru dans le $n^{\circ} 24$ (décembre 2000). La Libre Essentielle est un supplément du quotidien La Libre Belgique. Ce supplément mêle inextricablement la publicité à diverses rubriques de culture et de loisirs. Ce numéro présente, sur le thème général du «Chocolat », des vêtements et accessoires de couleur ... chocolat, dans le décor d'une chocolaterie. 
12. Sommes-nous ici face à un dessin d'humour ou à une publicité ? L'interprétation reste ouverte, puisque la marque Côte d'Or apparaît nettement sur l'image, accompagnée de son emblème représentant un éléphant.

\section{RÉSUMÉS}

Dans la première partie de ce travail, nous constaterons que la lexicologie a parfois considéré les collocations comme un phénomène particulier devant être étudié à part, après l'étude des unités lexicales « régulières".

En nous inspirant de la conception peircienne du signe, nous soutiendrons que ces expressions devraient au contraire être prises en compte dès le départ.

Nous prendrons un exemple concret (les expressions contenant le mot noir) pour illustrer notre démarche et nous montrerons comment le linguiste pourrait tirer parti de concepts tels que l'objet immédiat vs l'objet dynamique, le representamen et l'interprétant. Dans cette approche lexicale, des données jugées le plus souvent secondaires apparaissent au contraire importantes, et la notion peircienne d'interprétant est essentielle.

In the first part of this paper, we maintain that lexicology most often has considered collocations as a particular phenomenon that has to be studied separately on its own, after the "normal" lexical items.

In the second part we draw inspiration from the peircean theory of the sign for making the hypothesis that collocations have to be taken in account at the start. We will take one concrete example (the various expressions with the word noir) and we will show how concepts like immediate object vs dynamic object, representamen and interpretant can be used by a linguist. In our lexical approach often-dismissed lexical items became important, and the peircean notion of interpretant is essential.

\section{AUTEURS}

\section{NICOLE EVERAERT-DESMEDT}

Facultés universitaires Saint-Louis, everaert@fusl.ac.be

\section{GUY EVERAERT}

Haute Ecole Léonard de Vinci, encbw@skynet.be 